\title{
Paleobotánica del Triásico Superior del valle del río Biobío, Chile: Clase Filicopsida
}

Marcelo Leppe

Philippe Moisan

Eduardo Abad

Sylvia Palma-Heldt
Departamento de Botánica, Facultad de Ciencias Naturales y Oceanográficas, Universidad de Concepción, Casilla 160-C, Concepción, Chile Dirección actual: Departamento Científico, Instituto Antártico Chileno, Plaza Muñoz Gamero 1055, Punta Arenas, Chile mleppe@inach.cl

\author{
Facultad de Ciencias Naturales y Oceanográficas, \\ Universidad de Concepción, Casilla 160-C, Concepción, Chile \\ fmoisan@udec.cl.
}

Departamento de Ciencias de la Tierra, Universidad de Concepción, Chile eabad@udec.cl. sypalma@udec.cl

\section{RESUMEN}

Se describen restos fósiles de la Clase Filicopsida presentes en los estratos marinos y continentales del valle inferior del río Biobío $\left(37^{\circ} \mathrm{S} / 73^{\circ} \mathrm{W}\right.$ ) denominados formación Santa Juana y asignados al Triásico Superior (Cárnico-Nórico) de la Región del Biobío, Chile. La tafoflora incluye diez especies, de las cuales ocho son primeros registros para el Triásico de esta región: Gleichenites quilacoyensis Leppe y Moisan sp. nov., Asterotheca rigbyana rigbyana Herbst, Rienitsia colliveri Herbst, Dictyophyllum fuenzalidai Herbst, Dictyophyllum (Thaumatopteris) rothi Frenguelli, Chansitheca argentina Herbst, Cladophlebis kurtzi Frenguelli y Todites baldoni Herbst; tres son nuevos registros para Chile: Rienitsia colliveri Herbst, Gleichenites quilacoyensis Leppe y Moisan sp. nov. y Chansitheca argentina Herbst. Las especies de helechos encontrados representan el $18 \%$ de la diversidad específica total de plantas del Triásico del Biobío y ayudan a clarificar el complicado esquema de la evolución florística de las comunidades del Gondwana. Se sitúa al Triásico del Biobío en el contexto del Gondwana Extratropical Suroeste. Los conjuntos se desarrollaron en un ambiente continental de fuerte influencia marina, bajo un clima estacional lluvioso y con afinidad etánea con el estadio Floriano argentino (Nórico-Rético). Desde un enfoque evolutivo, se enmarca dentro del largo proceso de extinción masiva Triásico/ Jurásico, que significó el fin de la 'Flora de Dicroidium' y la aparición de una serie de elementos que dominarán durante el Jurásico.

Palabras claves: Triásico Superior, Paleobotánica, Filicópsidas, Chile.

\section{ABSTRACT}

Upper Triassic Palaeobotany of Biobío river valley, Chile: Filicopsida Class. The Upper Triassic (Carnian-Norian) Filicopsida fossils present in the marine and continental outcrops, informally nominated as Santa Juana formation in the Biobío Region of Chile $\left(37^{\circ} \mathrm{S} / 73^{\circ} \mathrm{S}\right)$, are described. The taphoflora includes ten species, out of which eight are first registers for this area: Gleichenites quilacoyensis Leppe and Moisan sp. nov., Asterotheca rigbyana rigbyana Herbst, Rienitsia colliveri Herbst, Dictyophyllum fuenzalidai Herbst, Dictyophyllum (Thaumatopteris) rothi Frenguelli, Chansitheca argentina Herbst, Cladophlebis kurtzi Frenguelli and Todites baldoni Herbst ; three are new records for the Chilean Upper Triassic: Rienitsia colliveri Herbst, Gleichenites quilacoyensis Leppe and Moisan sp. nov., and 
Chansitheca argentina Herbst. The fern species found represents an $18 \%$ of the total specific diversity of plants in the Upper Triassic rocks from Biobío and help to understand the complicate floristic evolution of the communities in Gondwana. The Triassic rocks from Biobío are situated in the context of the Southwestern Extratropical Gondwana. The assemblage was developed in a continental environment with strong marine influence, under a rainy seasonal weather and displays age affinities with the argentinian Florian stage (Norian-Rhaetian). On an evolutionary point of view, the paleoflora was involved into the long Triassic/Jurassic mass extinction process, that means the end of the 'Dicroidium Flora' and the start of several elements that will dominate during the Jurassic.

Key words: Upper Triassic, Palaeobotany, Filicopsida, Chile.

\section{INTRODUCCIÓN}

El Triásico se caracteriza a escala mundial por fuertes recambios florísticos. En primer lugar, el período comenzó con la mayor extinción masiva de la historia natural: la del límite Pérmico/Triásico (P/ T), que significó en el Gondwana el reemplazo de la 'Flora de Glossopteris' por la 'Flora de Dicroidium' (Balme y Helby, 1973). Sin embargo, estos activos lapsos de extinción masiva se caracterizan por afectar diferencialmente a los ambientes acuáticos y terrestres (Hallam y Wignall, 1997), así como por representar oportunidades para la radiación adaptativa de taxones suprimidos o de poco desarrollo en el pasado, lo que frecuentemente se ha usado como argumento a favor de la 'Teoría del Equilibrio Puntuado' (Eldredge y Gould, 1988). Por otro lado, el Triásico también emerge como un período de mejores condiciones ambientales que el Pérmico, para el desarrollo de nuevos linajes de plantas. En el Triásico gondwánico desaparecen las grandes masas de hielo que caracterizaron a las postrimerías del Pérmico, y que ocupaban gran parte de la superficie del megacontinente austral, dando origen a un clima de temperatura media $6^{\circ} \mathrm{C}$ más cálido que el Pérmico (Holser et al., 1991; Ziegler et al., 1994). En cambio, no existe la misma claridad respecto del evento que puso fin al Triásico, ya que como señalaron Hallam y Wignall (1997), la extinción a fines del Triásico fue temporalmente extensa, a diferencia de los otros eventos de extinción masiva del Mesozoico y, en el contexto regional, podría explicar la paulatina disminución de la dominancia de los Dicroidium desde el Cárnico superior, hasta hacerse totalmente subordinados en las floras réticas sudamericanas. Diversos autores han destacado que el registro de plantas terrestres se vio menos afectado que el registro de invertebrados marinos en el límite Triásico/Jurásico (Knoll, 1984; Ash, 1986; Traverse, 1988). Sin embargo, en una perspectiva cronológica más amplia, se observa que muchos cambios involucraron a los helechos con semillas desde antes del límite $T / J$. Las Glossopteridaceae se extinguieron completamente, así como las Corystospermaceae (que tenían una fuerte representación en las floras triásicas con los géneros Dicroidium, Xylopteris, Johnstonia y Zuberia) y las Peltaspermaceae decayeron fuertemente (Hallam y Wignall, 1997).

En el Triásico gondwánico, como ya se ha dicho, las filicópsidas se encuentran principalmente representadas por dos grupos: uno de taxones relativamente primitivos, procedentes del Paleozoico y un segundo, más moderno, que radiaría a partir de mediados del Triásico (Zamuner et al., 2001). Frecuentemente, se considera a las familias de leptosporangiadas las más primitivas de todas las Filicales, a pesar de que Stewart (1983), señaló que las leptosporangiadas evolucionaron a partir de las euesporangiadas como las Osmundaceae y Gleicheniaceae, que se encuentran bien representadas en muchos afloramientos triásicos gondwánicos (Stewart, 1983; Zamuner et al., 2001). En cambio, otros importantes grupos modernos, como Dicksoniaceae, Cyathiaceae, Mationiaceae, Polypodiaceae y Dipteridaceae hallan su génesis en el Triásico.

Con relación a las edades, en este trabajo se utiliza la escala de tiempo geológico de Gradstein et al. (2004). 


\section{TRIÁSICO DEL BIOBÍO Y CONTEXTO REGIONAL}

El dominio Triásico gondwánico se compone de dos áreas: una tropical y una extratropical (Spalletti et al., 2003) (Fig. texto 1). El área extratropical se define palinológicamente por la microflora de 'Ipswich' y por la megaflora de 'Dicroidium' que se caracterizan por una alta exclusividad (Anderson y Anderson, 1983; Artabe et al., 2002). Desde el Triásico Medio y hasta la mitad del Triásico Tardío (Estadio Cortaderitiano) comienzan a cambiar nuevamente las condiciones abióticas que se han de manifestar en nuevas asociaciones florísticas. A comienzos del Triásico Tardío ya se ha establecido un régimen subtropical seco, fuertemente estacional y que configura un ambiente de carácter semiárido (Tanner, 2000; Spalletti et al., 2003). Florísticamente este lapso se caracteriza por la máxima diversificación de la 'Flora de Dicroidium' (Spalletti et al., 1999; Zamuner et al., 2001; Spalletti et al., 2005), con bosques estacionales subtropicales siempreverdes dominados por Corystospermaceae, Voltziaceae y Protopinaceae (Spalletti et al., 2003). Hacia el Triásico Tardío se reconocen nuevos cambios en el ambiente físico que condicionarán la existencia de nuevas paleocomunidades. Durante el lapso se produjo una activación de la tectónica al oeste delárea extratropical (Chile y oeste argentino), muy cerca del borde costero Protopacífico, formándose 'rifts' extensionales angostos y aislados, con un volcanismo asociado que generó abundantes flujos de lava y depósitos volcanoclásticos que, al menos localmente, evidencian asociaciones metamórficas indicativas de condiciones de muy bajo grado (Charrier, 1979; Franzese y Spalletti, 2001; Spalletti et al., 2003; Suárez y Bell, 1992; Belmar y Morata, 2005). Al oeste se reconoce la formación de depósitos continentales y marinos, y al este hemigrábenes de sedimentación netamente continental. Un régimen de precipitaciones megamonzónicas se estableció en el margen oeste, produciendo una gradual acentuación de la aridez hacia el interior del continente. Este incremento en la humedad se debió a un desplazamiento latitudinal del Gondwana, que durante el Triásico Temprano permaneció relativamente constante (Tanner, 2000). El lapso Cárnico superior-Rético se caracteriza florísticamente por la decreciente presencia de Corystospermaceae, aparición de elementos típicos del Jurásico, dominancia de bosques estacionales decíduos con el estrato arbóreo dominado por Voltziaceae y Ginkgoales en los

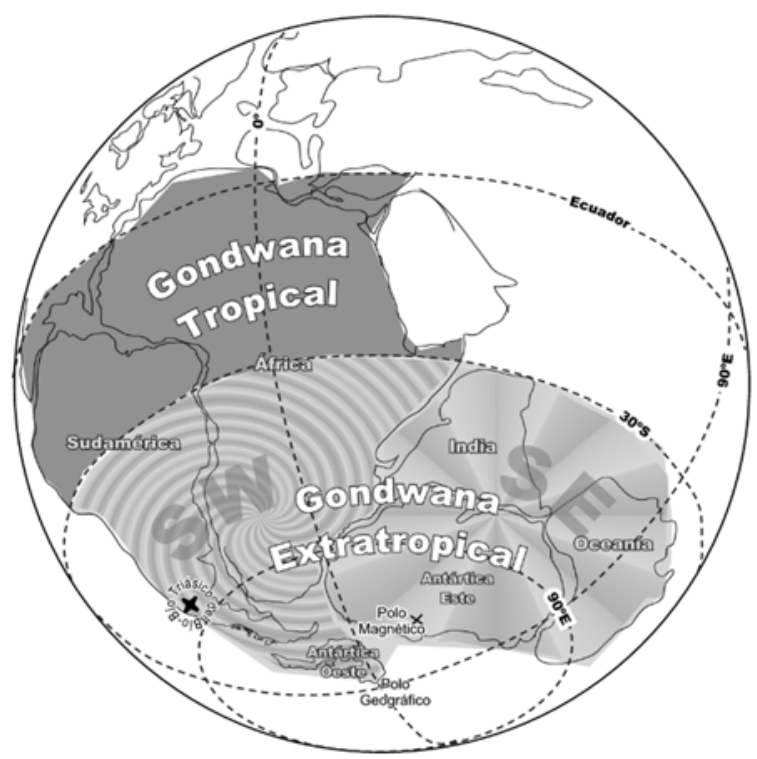

FIG. texto 1. Mapa de las dos zonas del Gondwana durante el Triásico Superior; se destaca la zona del Gondwana Extratropical donde se ubican los afloramientos triásicos del Biobío (modificado de Spalletti et al., 2003. 


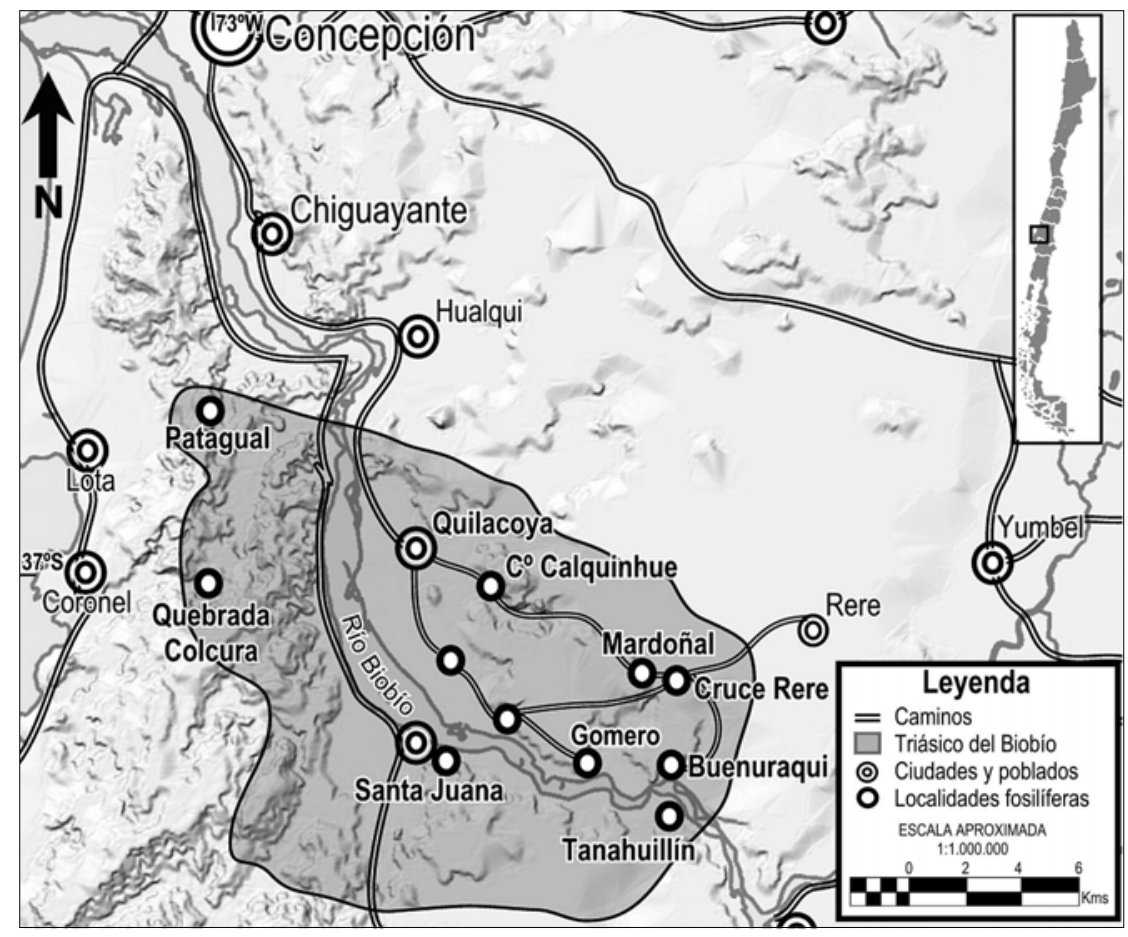

FIG. texto 2. Mapa de las dos zonas del Gondwana durante el Triásico Superior, destacándose la zona del Gondwana Extratropical donde se ubican los afloramientos triásicos del Biobío.

ambientes interiores, y por Linguifolium en los ambientes de influencia marina (Retallack, 1987; Artabe et al., 2001; Leppe y Moisan, 2003; Spalletti et al., 2003).

Es en este marco, entonces, en el que se insertan los afloramientos del valle inferior del río Biobío (Fig. texto 2). Existen varias evidencias que sitúan a estos estratos en los segundos dos tercios del Triásico Tardío: en primer lugar, la sensible baja frecuencia de Corystospermaceae (Leppe et al., 2003) y sólo relegada al sotobosque; en segundo lugar, la actividad volcánica que caracteriza al lapso, estaría registrada extensamente en el área de Cerro Calquinhue en la ribera norte del río Biobío (Leppe et al., 2003); y el establecimiento de un bosque deciduo subtropical oceánico con una fuerte presencia en el estrato arbóreo de Linguifolium. Lo anterior situaría al Triásico del Biobío entre el Cárnico superior y el Nórico, lo que se corroboraría por el reciente registro de artrópodos fósiles (Gallego et al., 2005).

\section{FILICÓPSIDAS EN EL TRIÁSICO CHILENO}

Se ha caracterizado al Triásico como un período en el que los helechos son una mezcla de grupos primitivos provenientes del Paleozoico y de otros modernos que se diversifican durante el Triásico Tardío (Zamuner et al., 2001). Algunos helechos como Cladophlebis, Asterotheca, Chansitheca, Gleichenites y Coniopteris exhibieron una distribución ecuménica. Géneros como Asterotheca y Chansitheca tuvieron su acmé durante el Pérmico y han permanecido como remanentes en el Triásico. Otros géneros como Rienitsia han aparecido y se han extinguido durante el Triásico. En cambio, grupos como las Gleicheniaceae hallan su origen en el Neopaleozoico, con una reducida representación en el Triásico, y su mayor diversificación durante el Cretácico Temprano (Wang et al., 1999). Las Dicksoniaceae, por otra parte, se originan en el Triásico Temprano y tiene su máxima expresión entre el Jurásico y Cretácico. Por otro lado, algunas 
Dipteridaceae, como Dictyophyllum, son elementos típicos de la flora triásica que persisten durante el Jurásico.

En Chile centro sur el estudio de filicópsidas ha sido escaso, a diferencia del norte, donde destacan en La Ternera (Región de Atacama) los trabajos paleobotánicos de Herbst et al. (1998), Troncoso y Herbst (1999) y Herbst (1996, 2000), que reportan las filicópsidas Dictyophyllum fuenzalidai Herbst, $D$. tenuifolium Bonetti y Herbst, $D$. rothi Frenguelli y Goeppertella taverai Herbst entre las Dipteridaceae; Gleichenites cachivaritensis Herbst, una Gleicheniaceae de Quebrada la Cachivarita; Rienitsia ternerae Herbst, Asterotheca rigbyana Herbst y $A$. fuchsi Herbst entre las Asterothecaceae; Todites chilensis Herbst y Troncoso, Cladophlebis mendozaensis Frenguelli y $C$. kurtzi Frenguelli que representan a las Osmundaceae.

En el Triásico del Biobío, Steinmann (1921) reconoció una serie de vegetales fósiles de edad rética, entre los que destaca la filical Clathropteris platyphylla Goeppert (Tavera, 1960). En esta misma región, en la localidad de Pocillas (noroeste de la Provincia de Ñuble) se han descrito depósitos con ejemplares de Taeniopteris mareyesiaca Oishi y Dicroidium lancifolium Gothan (Hervé et al., 1976), que se asemejan mucho a los afloramientos de La Ternera.

Éste es el contexto en que surgen durante el Triásico Tardío las asociaciones florísticas de Chile centro sur, donde destacan los afloramientos denominados informalmente como formación Santa Juana (Tavera, 1960; Cucurella, 1978; Tanai, 1981).

Existe una gran diversidad específica en los afloramientos triásicos de Chile y Argentina (Fig. texto 3). Charrier (1979) destacó entre los morfogéneros de filicópsidas presentes en Chile a Asterotheca, Chansitheca, Cladophlebis, Dictyophyllum. Sin embargo, más de la mitad de la diversidad de filicópsidas chilenas ha sido informada en los afloramientos de la Formación La Ternera. En la Provincia de Petorca, se han descrito sucesiones triásicas plantíferas en Los Vilos-Caleta los Molles (Corvalán, 1976; Azcárate y Fasola, 1970), así como en La Ligua (Provincia de Aconcagua), Gualleco, Estero Oñolco y Cuesta EI Culebrón (Provincia de Talca) con Clathropteris platyphylla Brongniart (Thiele, 1965). Los autores han podido recolectar material fragmentario de Dictyophyllum tenuifolium y Asterotheca sp. en afloramientos ubicados en Curepto y Hualañé en la

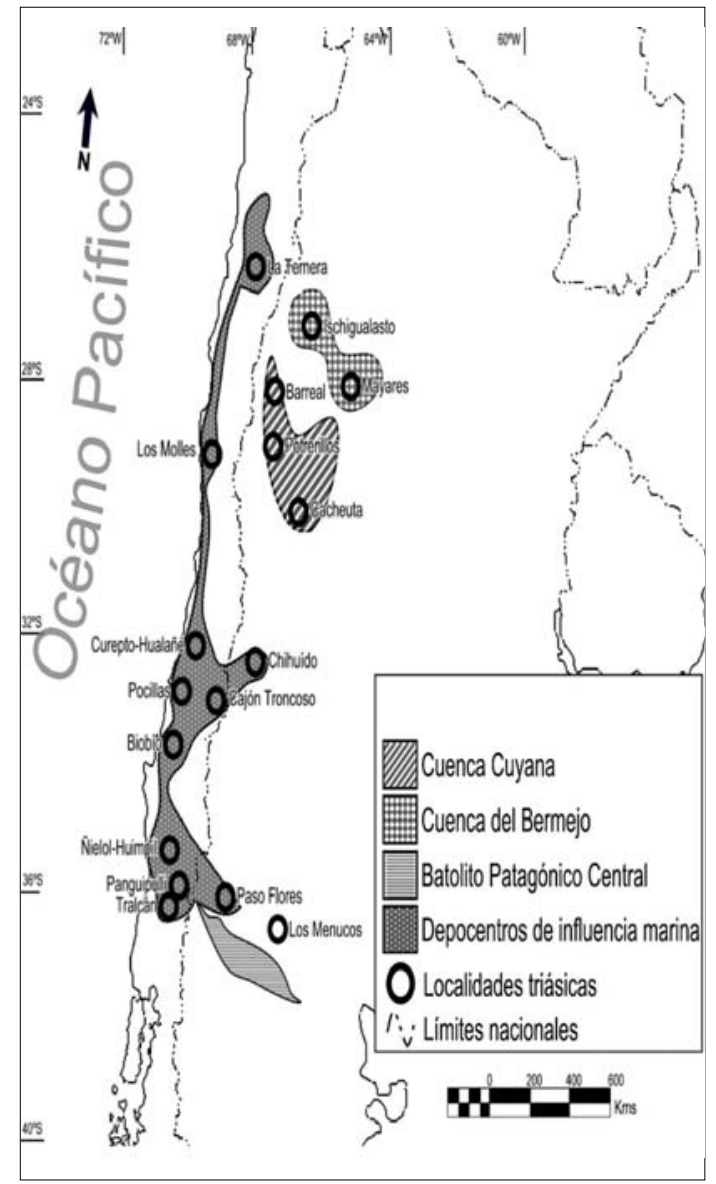

FIG. texto 3. Mapa de las principales localidades triásicas de Chile y Argentina.

VI y VII regiones, todos afloramientos asignados al Triásico Tardío (Corvalán, 1976; Charrier, 1979) . En Temuco, se reconocen secuencias triásicas desde Río Quillén hasta Cerro Humbulli, probablemente de origen continental y con gran similitud litológica con el valle del río Biobío, pero ciertamente muy poco estudiados (Hervé et al., 1976). La localidad principal está ubicada en Valle Huimpil (Corvalán, 1976; Tanai, 1981) donde se ha observado también la presencia de Gleichenites sp. en material recolectado por R. Herbst y A. Troncoso. En el cerro Tralcán (oeste del lago Riñihue) se han descrito afloramientos con abundantes improntas de filicópsidas, tales como Cladophlebis (Hervé et al., 1976; Zavattieri et al., 2003). Otra localidad triásica que presenta filicópsidas es la Formación Panguipulli que agrupa los depósitos triásicos del lago del mismo nombre, 
del margen norte del lago Calafquén e Isla Huapi en el lago Ranco, todos en la Región de Los Lagos
(Thiele et al., 1976; Arrondo et al., 1988).

\section{MATERIALES Y MÉTODOS}

El material estudiado corresponde a improntas de hojas procedentes de distintas localidades del valle inferior del río Biobío (Fig. texto 2) recolectadas durante varias campañas en el marco de los proyectos Fondecyt 2010105 y DIUC 99025010-10, y durante el curso Geología de Campo Il a cargo de los docentes Arturo Quinzio y Ramiro Bonilla del Departamento Ciencias de la Tierra de la Universidad de Concepción. Los sitios estudiados se reseñan en la tabla 1 . El material recolectado fue depositado en el Museo del Departamento de Ciencias de la Tierra (CONC) de la Universidad de Concepción, donde fue descrito mediante el uso de una lupa Carl Zeiss y una cámara clara para la realización de diagramas. Las fotografías (60) se obtuvieron mediante una cámara digital Sony Mavica con un 'zoom' de 30x. Las improntas quedaron alojadas y etiquetadas como STJU 1-62 en el estante identificado como 'Triásico y Paleozoico' de las Colecciones Paleontológicas de la Universidad de Concepción. El holotipo de la especie nueva Gleinchenites quilacoyensis se encuentra alojado con el códico STJU en la Colección del Laboratorio de Paleobotánica del Departamento Ciencias de la Tierra, Universidad de Concepción.

\section{ESTRATIGRAFÍA}

A estas rocas estratificadas se les ha dado informalmente varios nombres de unidades litoestratigráficas, como el de Formación Santa Juana y otros, pero no existe una proposición formal, ni una sección estratigráfica tipo, por lo que es conveniente continuar con la denominación de 'Triásico del valle inferior del Biobío', como las denominó Brüggen (1950).

La estratigrafía de los afloramientos de rocas triásicas del valle del Biobío corresponde a secuencias sedimentarias constituidas por arcosas y litarenitas feldespáticas, con intercalaciones acuñadas de lutitas negras y presencia subordinada de ruditas. Existen, además, en un par de localidades restringidas, intercalaciones de rocas volcánicas que alcanzan los $500 \mathrm{~m}$ de espesor, correspondientes a andesitas y tobas, informadas, por primera vez, por Abad y Figueroa (2003). Las potencias de estas sucesiones sedimentarias varían desde 10 a $700 \mathrm{~m}$ de acuerdo a la continuidad de los afloramientos, dispuestos discordantemente sobre rocas metamórficas y granitoides del Paleozoico. Su techo no aflora en el área (Fig. texto 4).

Las arcosas y litarenitas feldespáticas son de granulometría muy variada encontrándose desde muy gruesas a muy finas en estructura maciza, principalmente, y con buena estratificación sólo en algunas localidades con estratos de 10 a $70 \mathrm{~cm}$. Se observan estructuras de estratificación cruzada, laminación cruzada, laminación plana y 'ripple marks', en ambiente lacustre.

Las intercalaciones de lutitas son fisibles, de variados espesores desde $1 \mathrm{~cm}$ a $10 \mathrm{~m}$ y extensiones laterales de centímetros a decenas de metros, donde la geometría acuñada es muy común, de color gris oscuro a negro, que representan probables llanuras de inundación, canales abandonados y superficies de 'ripple marks'. Son estas lutitas las que poseen fundamentalmente el contenido orgánico y paleontológico. En algunos sectores son altamente carbonosas, por lo que en el pasado hubo pequeñas explotaciones de carbón.

Las ruditas son variadas, desde un conglomerado fino cuarcífero clasto soportado, de un par de metros de potencia y ocupa posiciones basales en ciertos sectores, a otro brechoso, macizo, con clastos centimétricos y decimétricos con niveles clasto soportados y otros matriz soportados, de potencia aproximada a los $200 \mathrm{~m}$, a varios de geometría acuñada de clastos centimétricos, clasto soportados, en las arenitas. En horizontes de areniscas macizas, de potencias superiores a tres metros se aprecian niveles congloméradicos de contactos difusos. En general, los clastos son de rocas metamórficas y graníticas, las que conforman el zócalo de las rocas triásicas.

La asociación de facies general del área sugiere un medio aluvial muy cercano a un borde costero (Abad, 2000), con cierta influencia marina, comprobada por la presencia de abundante fauna de invertebrados (que incluye nautiloideos, indicadores de ambiente de influencia oceánica) y vegetales 
TABLA 1. LOCALIDADES TRIÁSICAS DEL VALLE INFERIOR DEL RÍO BIOBÍO DONDE SE HALLARON LOS EJEMPLARES STJU 1-62, CON SUS COORDENADAS GEOGRÁFICAS Y NÚMERO ROMANO CORRELATIVO.

\begin{tabular}{|c|c|c|c|}
\hline \multicolumn{2}{|c|}{ Localidades } & \multicolumn{2}{|c|}{ Coordenadas } \\
\hline I & Santa Juana, bajada al río desde la plaza & $37^{\circ} 01.307^{\prime} \mathrm{S}$ & $73^{\circ} 01.300^{\prime} \mathrm{W}$ \\
\hline II & Buenuraqui, vía férrea hacia Gomero & $37^{\circ} 12.138^{\prime} \mathrm{S}$ & $72^{\circ} 47.665^{\prime} \mathrm{W}$ \\
\hline III & Santa Juana, camino forestal & $37^{\circ} 13.630^{\prime} S$ & $72^{\circ} 49.601$ 'W \\
\hline IV & Camino a Quilacoya 1 & $37^{\circ} 03.395^{\prime} \mathrm{S}$ & $72^{\circ} 52.305^{\prime} \mathrm{W}$ \\
\hline V & Camino a Quilacoya desde Chillancito 1 & $37^{\circ} 04.234^{\prime} S$ & $72^{\circ} 49.260^{\prime} \mathrm{W}$ \\
\hline $\mathrm{VI}$ & Camino a Quilacoya desde Chillancito 1 & $37^{\circ} 04.700^{\prime} S$ & $72^{\circ} 56.330^{\prime} \mathrm{W}$ \\
\hline VII & Cerro Calquinhue & $37^{\circ} 05.955^{\prime} \mathrm{S}$ & $72^{\circ} 53.534^{\prime} \mathrm{W}$ \\
\hline VIII. & Gomero, camino a Buenuraqui & $37^{\circ} 12.223^{\prime} \mathrm{S}$ & $72^{\circ} 47.890^{\prime} \mathrm{W}$ \\
\hline IX & Patagual 1 & $37^{\circ} 01.300^{\prime} \mathrm{S}$ & $73^{\circ} 01.200^{\prime} \mathrm{W}$ \\
\hline $\mathrm{X}$ & Patagual 2 & $37^{\circ} 01.628^{\prime} S$ & $73^{\circ} 16.940^{\prime} \mathrm{W}$ \\
\hline $\mathrm{XI}$ & Mardoñal & $37^{\circ} 07.011^{\prime} \mathrm{S}$ & $72^{\circ} 50.220^{\prime} \mathrm{W}$ \\
\hline XII & Cruce Rere & $37^{\circ} 08.105^{\prime S}$ & $72^{\circ} 50.106^{\prime} \mathrm{W}$ \\
\hline XIII & Talcamávida & $37^{\circ} 08.205^{\prime S}$ & $72^{\circ} 54.080^{\prime} \mathrm{W}$ \\
\hline XIV & Gomero & $37^{\circ} 11.302^{\prime} \mathrm{S}$ & $72^{\circ} 50.231^{\prime} \mathrm{W}$ \\
\hline$X V$ & Buenuraqui & $37^{\circ} 11.602^{\prime} \mathrm{S}$ & $72^{\circ} 47.403^{\prime} \mathrm{W}$ \\
\hline $\mathrm{XVI}$ & Quebrada Colcura & $37^{\circ} 11.556^{\prime} S$ & $73^{\circ} 16.024^{\prime} \mathrm{W}$ \\
\hline XVII & Santa Juana, 'by pass' & $37^{\circ} 10.270^{\prime} S$ & $72^{\circ} 57.406^{\prime} \mathrm{W}$ \\
\hline
\end{tabular}

como Linguifolium (Leppe y Moisan, 2003).

El área estudiada está afectada por una intensa tectónica y fuerte erosión que influye en la distribu- ción de los afloramientos y modelado del paisaje, lo que dificulta las correlaciones.

\title{
DESCRIPCIONES SISTEMÁTICAS
}

\author{
Clase Filicopsida Cleal y Thomas, 1994 \\ Orden Marattiales Prantl, 1874 \\ Familia Asterothecaceae Cleal y Thomas, 1994 \\ Género Asterotheca Presl in Corda, 1845
}

Especie tipo: A. sternbergii (Göpp.) Presl in Corda, 1845. Carbonífero de Saarbrücken, Alemania.

\section{Asterotheca rigbyana rigbyana Herbst, 1977a}

Lám. 1, Figs. 1-2; Lám. 4, Fig. 16

Material estudiado: STJU No. $04,07,13,18,25$ y 27.

Procedencia geográfica: Cerro Calquinhue, camino a Buenuraqui, Provincia de Concepción, Región del Biobío (Fig. texto 2; Tabla 1).

Discusión: originalmente, el género Asterotheca fue descrito por Presl para el Carbonífero de Alemania (Corda, 1845). Sin embargo, su presencia se prolonga hasta el Neotriásico, donde exhiben una marcada exclusividad en el sur de Sudamérica
(Zamuner et al., 2001). Los caracteres diagnósticos del material estudiado coinciden con los ejemplares descritos por Herbst (1977a) y Herbst et al. (1998), salvo en detalles como el tamaño de las pinnas y el largo de las pínnulas, un poco más largas en el caso de las aquí estudiadas. A. rigbyana rigbyana aparece como una de las subespecies típicas del Triásico Superior de Argentina. Ha sido reconocida en la Formación Paso Flores, Neuquén (Morel et al., 2000) para el Triásico Superior y en la Formación Cañadón Largo, Provincia de Santa Cruz, Argentina (Herbst, 1988). En Chile, se registra en la Formación La Ternera, Provincia de Copiapó (Herbst et al., 1998), también para el Triásico Superior. Éste es el primer registro de $A$. rigbyana rigbyana para el Triásico del Biobío. 


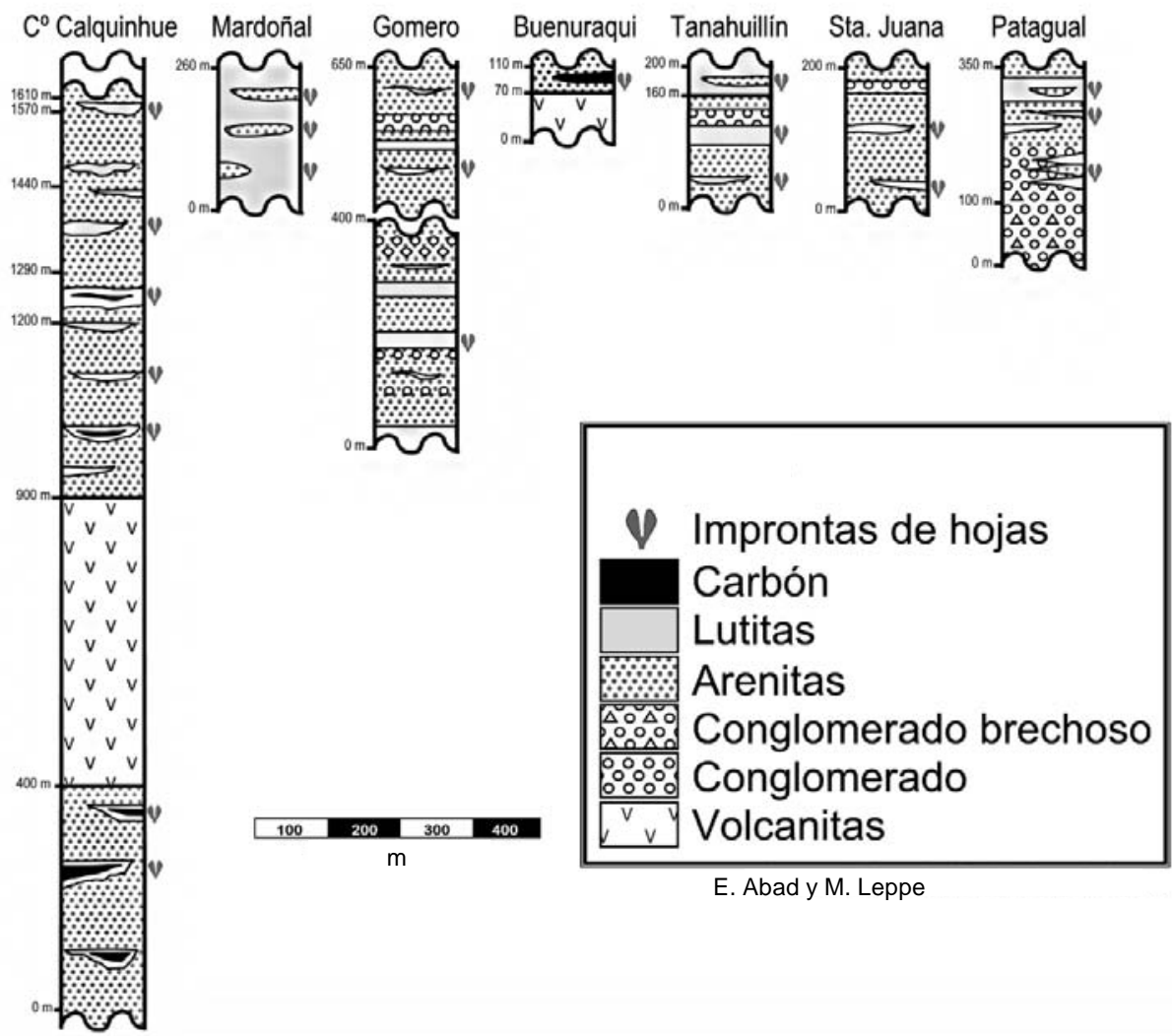

FIG. texto 4. Columnas estratigráficas de las localidades estudiadas en los afloramientos triásicos, valle inferior del río Biobío.

Asterotheca fuchsi (Zeiller) Herbst, 1998 Lám. 1, Fig. 3; Lám. 4, Fig. 17

Material estudiado: STJU No. 06, 29 y 39. Procedencia geográfica: Patagual y Cerro Calquinhue, Provincia de Concepción, Región del Biobío (Fig. texto 2; Tabla 1).

Discusión: esta especie se encuentra exclusivamente representada en rocas del Triásico Superior del sur de Sudamérica. El material se ajusta bien con el descrito por Herbst et al. (1998) para la tafoflora de La Ternera, pero dichos autores señalaron un número de 12 o más sinangios por hemilámina y un tamaño sensiblemente menor para las pínnulas (13 $\mathrm{mm}$ de largo en el caso del material de La Ternera y hasta $20 \mathrm{~mm}$ de largo para el material del Triásico del Biobío). Los demás caracteres coinciden con el material estudiado.
Género Rienitsia Walkom, 1932

Especie tipo: R. spathulata Walkom, 1932. Mesotriásico-Neotriásico de las proximidades del monte Victoria, Australia.

\section{Rienitsia colliveri Herbst, 1977b \\ Lám. 1, Fig. 4; Lám. 4, Fig. 18}

Material estudiado: STJU No. 16, 40.

Procedencia geográfica: Cerro Calquinhue, Patagual y Mardoñal, Provincia de Concepción (Fig. texto 2; Tabla 1).

Discusión: la especie Rienitsia colliveri ha sido asignada al Triásico Superior (Herbst , 1977). El material estudiado coincide con la diagnosis de Herbst (1977b), ya que presenta ala laminar a lo largo del raquis, y concuerda plenamente con los 
caracteres dados para la fronda estéril. A pesar de carecer de una fronda fértil para esta especie en el Triásico del Biobío, los caracteres que aquí se mencionan permiten con certeza la asignación especifica. La otra especie, descrita para Chile es Rienitsia ternerae (Herbst et al., 1998), que coincide parcialmente en los tamaños con el material aquí estudiado, como por ejemplo, el ancho del raquis de la pinna que es considerablemente mayor en la especie $R$. ternerae.

No existen otras especies de Rienitsia descritas para Chile, y éste es el primer registro del género para el Triásico del Biobío.

\section{Orden Filicales Dumortier, 1829 \\ Familia Dipteridaceae (Diels) Seward y Dale, 1901 \\ Género Dictyophyllum (Lindley y Hutton, 1834); Webb, 1982 \\ Subgénero Dictyophyllum (Dictyophyllum) Herbst, 1992}

Especie tipo: D. rugosum Lindlley y Hutton, 1834. Jurásico de Yorkshire, Inglaterra.

Dictyophyllum (Dictyophyllum) tenuifolium (Stipanicic y Menéndez) Bonetti y Herbst, 1964 Lám. 1, Fig. 5; Lám. 4, Fig. 19

Material estudiado: STJU Nos. 11, 12, 15, 21, 26, 55, 56 y 57.

Procedencia geográfica: Santa Juana, vía férrea hacia Gomero, camino a Quilacoya, Cerro Calquinhue, Patagual, Mardoñal y Quebrada Colcura, Provincia de Concepción, Región del Biobío (Fig. texto 2; Tabla 1). Discusión: el biocrón de esta especie la sitúa en el Triásico Superior (Herbst, 1992). En ciertos niveles del Triásico del Biobío, Dictyophyllum tenuifolium es tan abundante que forma asociaciones casi puras, como es el caso de Cerro Calquinhue y Tanahuillín. El material encontrado se ajusta muy bien a la diagnosis de Bonetti y Herbst (1964), con frondas relativamente completas y detalle de la venación muy bien preservada. La especie ha sido hallada en el Cárnico de la Formación Barreal (Stipanicic y Menéndez, 1949) y Formación Paso Flores (Bonetti y Herbst, 1964) en Argentina, así como en la Formación La Ternera (Herbst et al., 1998), en Chile, coincidiendo para los afloramientos citados la edad triásica superior.

\section{Dictyophyllum (Dictyophyllum) fuenzalidai Herbst, 2000 \\ Lám. 2, Fig. 8; Lám. 4, Fig. 20}

Material estudiado: STJU No. 60.

Procedencia geográfica: Santa Juana, Provincia de Concepción, Región del Biobío (Fig. texto 2, Tabla 1).

Discusión: taxón de ocurrencia muy rara en el Triásico del Biobío (Cerro Calquinhue). A pesar de la ausencia de venación de orden mayor, presenta suficientes características que permiten asignar este ejemplar a la especie de la Formación La Ternera, en el norte de Chile, único lugar donde se ha registrado (Herbst, 2000).

\section{Subgénero Dictyophyllum (Thaumatopteris) Herbst, 1992 \\ Dictyophyllum (Thaumatopteris) rothi Frenguelli, 1941}

Lám. 2, Figs. 6-7; Lám. 4, Fig. 21

Material estudiado: STJU No. 10, 50, 58 y 59. Procedencia geográfica: Buenuraqui, Talcamávida, Gomero y Cruce Rere, Provincia de Concepción, Región del Biobío (Fig. texto 2, Tabla 1).

Discusión: en Argentina fue descrita originalmente por Frenguelli (1941) para el Liásico de Mendoza y
Neuquén. Posteriormente, Arrondo et al. (1991) reportó el hallazgo de $D$. rothi en la Formación Paso Flores, lo que extiende su distribución desde el Triásico Superior al Jurásico Inferior. La especie es de ocurrencia poco frecuente en el Triásico del Biobío (Cerro Calquinhue y Santa Juana). La diagnosis coincide bien con la enunciada por Herbst 
(1992, 2000); sin embargo, no existía información referencial de frondas fértiles en esta especie. Es el primer registro del taxón en el Triásico del Biobío, encontrado anteriormente en Chile en Quebrada

El Carbón, Formación La Ternera (Herbst, 2000).

Familia Gleicheniaceae Presl, 1845

Género Geichenites Seward, 1926

Especie tipo: Gleichenites porsildi Seward, 1926. Cretácico del oeste de Groenlandia.

\section{Gleichenites quilacoyensis Leppe y Moisan sp. nov.}

Lám. 2, Figs. 9-11; Lám. 5, Fig. 22.

Derivatio nominis: el epíteto específico hace referencia a la abundante presencia en ciertos estratos próximos a la localidad de Quilacoya, como los del cerro Calquinhue.

Procedencia geográfica: Cerro Calquinhue, Provincia de Concepción, Región del Biobío (Fig. texto 2; Tabla 1), Triásico Superior.

Material estudiado: Holotipo: STJU No. 2. Otros ejemplares estudiados: STJU Nos. 3 y 5.

Diagnosis: restos de frondas de forma y tamaño desconocidos, al menos bipinnadas, de $15-17 \mathrm{~cm}$ de largo por 10-12 cm de ancho. Raquis principal de 5,0 mm en su parte más ancha, y de 0,4-0,5 mm en su porción distal. Pinnas rectas, separadas, de 8,0 a 15,0 mm entre sí, dispuestas subalternamente a lo largo del raquis, que emergen en ángulos entre 45 y $50^{\circ}$. Pínnulas cladofleboides, ligeramente falcadas y unidas anchamente por la base en la porción apical de las frondas, pecioladas y redondeadas en la base de las pinnas. Ápice redondeado en todos los casos. Pínnulas de 5,0 a 7,0 mm de largo por 3,0-5,0 mm de ancho, de borde liso y dispuestas subalterna a subopuestas. Vena media conspicua y ligeramente tortuosa; venas secundarias levemente curvadas, que emergen en un número constante de seis por pínnula. Las primeras, segundas y cuartas venas secundarias presentan una dicotomía en su primer tercio, llegando todas al margen. Pínnulas fértiles con cinco sori por pínnula, dos en la hemilámina apical y tres en la hemilámina basal. Los sori son redondeados de 0,4 a 0,7 $\mathrm{mm}$ de ancho, que decrecen en tamaño hacia el ápice de la pínnula; penta esporangiados, con esporangios piriformes. Ubicación del anillo desconocida.
Diagnosis: fragmentary fronds, at least bipinnated, $15-17 \mathrm{~cm}$ long and $10-12 \mathrm{~cm}$ wide. Main rachis 5 $\mathrm{mm}$ wide in their largest part and $0.4-0.5 \mathrm{~mm}$ in the distal portion. Straight pinnae, with $8.0-15.0 \mathrm{~mm}$ between each one, subalternate, emerging in angles between 45 and $50^{\circ}$. Cladophleboid pinnules, slighly falcate and broadly united by their basis in the pinnules of the distal part (apical) of the pinnae and more petiolated in the proximal part (basal) of the pinnae. Apex, in all the situations, rounded. Pinnules of 5.0-7.0 mm long and 3.0-5.0 mm wide, smooth border and subopposite to subalternate arrangement. Middle vein conspicuous and slightly tortuous, secondary veins mildly curvate, emerging in a constant number of six per pinnule. The first, second and fourth secondary veins presents one dichotomy in the third part, reaching all to the margin. Fertile pinnules with five sori per pinnule, two in the apical hemilamina and three in the basal. The sori are rounded with 0.4 to $0.7 \mathrm{~mm}$ in diameter, decreasing in size to the apex of the pinnule. They are pentasporangiate with pyriform sporangia. Ubication of the annulus unknown.

Discusión: los especímenes del Triásico del Biobío comparten varias características con las especies Gleichenites gallegoi y G. cachivaritensis, como los sori pentaesporangiados, pero en los aquí descritos se observan cinco sori en todas las muestras, característica más afín con G. cachivaritensis, que en todo caso, según la diagnosis de Herbst (1996), presenta en su mayoría cuatro y ocasionalmente cinco. La venación de las pínnulas es muy regular en los especímenes estudiados y su arquitectura es idéntica en todos los ejemplares. Esta venación se diferencia notablemente de Gleichenites sp. descrita por Herbst y Troncoso (1996) para la Formación Chacarilla (Jurásico Medio del norte de Chile), sin dicotomías en las venas secundarias. El tamaño del material señalado como Gleichenites sp. por Herbst (1996) para el Miembro Talcamávida-Gomero (sensu Tavera, 1960, perteneciente al Triásico del Biobío) es comparable, 
pero en la venación secundaria ocasionalmente puede haber una vena basal furcada, lo que puede deberse al grado de preservación del material. Sin embargo, se destaca la presencia de frondas fértiles, con tres o cuatro sori por pínnula, diferentes de los cinco constantes en el material descrito aquí. Herbst (1996) en esa ocasión estableció varios nexos con G. gallegoi Herbst. Sin embargo, aún queda la posibilidad que el material descrito para Gomero por Herbst (1996) pertenezca al mismo taxón que el creado aquí, tanto por lo fragmentario del material, como por lo escaso del mismo. Con G. gallegoi se diferencia fundamentalmente porque la especie descrita por Herbst (1996) presenta 6-7 sori por pínnula y 4-7 esporangios por soro. En virtud de que los caracteres diagnósticos de esta nueva especie la sitúan muy cerca de las especies $G$. gallegoi y G. cachivaritensis, pero evidentemente por sus características propias no puede sino asignarse a una nueva especie.

\section{Género Chansitheca Regè, 1920}

Especie tipo: Chansitheca paleosilvana Regè, 1920. Carbonífero de Milán, Italia.

\section{Chansitheca argentina Herbst, 1963} Lám. 3, Fig. 12-13; Lám. 5, Fig. 23

Material estudiado: STJU No. 01, 41, 42, 43, 44 y 46.
Procedencia geográfica: Cerro Calquinhue, Gomero, camino a Buenuraqui, Provincia de Concepción, Región del Biobío (Fig. texto 2, Tabla 1).

Discusión: el material coincide plenamente con las características señaladas por Herbst (1963) para la especie Chansitheca argentina; sin embargo, las frondas fértiles tienen una gran similitud con Cladophlebis mendozaensis (Geinitz) Frenguelli, asunto que se discute más adelante. El género Chansitheca pertenece a un grupo de gleicheniáceas, en el cual también se agrupa Szea, remanentes del Paleozoico. Tidwell y Ash (1994) señalaron la presencia de Chansitheca en el Carbonífero de China, lo que convierte al taxón en el más antiguo de la familia. Sin embargo, otros autores como Yao y Taylor (1988) y Collinson (1996) pusieron en duda la pertenencia del género a la familia Gleicheniaceae. Incluso Herbst $(1963,1988)$ señaló, en una de las pocas relaciones que se tiene acerca de las frondas estériles, que podría ser atribuida a Cladophlebis en ausencia de caracteres fértiles. Resulta interesante destacar que la especie Chansitheca argentina es la única especie del género descrita para Argentina y para Sudamérica. Su presencia es sólo ocasional en los estratos del Grupo el Tranquilo, Provincia de Santa Cruz, Argentina, lo que convierte a este taxón en su primera cita para Chile.

\section{Familia Osmundaceae Brecht y Presl, 1820 Género Cladophlebis Brongniart, 1849}

Especie tipo: C. albertsii Brongniart, 1849. Carbonífero-Jurásico de Europa central.

\section{Cladophlebis kurtzi Frenguelli, 1947} Lám. 3, Fig.14; Lám. 5, Fig. 24

Material estudiado: STJU No. STJU 10, 37, 48 y 61. Procedencia geográfica: Santa Juana y Cerro Calquinhue, Provincia de Concepción, Región del Biobío (Fig. texto 2; Tabla 1).

Discusión: diversos autores han señalado la dificultad para diferenciar ciertas especies de Cladophlebis (Herbst, 1980; Herbst et al., 1998; Brea, 2000). En el caso del material aquí estudiado, se ha preferido adherir a la tendencia de diferenciar- las sobre la base de las características de la arquitectura pinnular señaladas por Brea (2000). Por lo anterior, se ha situado a este material en la especie C. kurtzi, que se diferencia con C. mendozaensis (Geinitz) Frenguelli en que esta última posee más de una dicotomía en la venación secundaria y una notable crenación de los bordes de las pínnulas. Con C. mesozoica se diferencia fundamentalmente por el tamaño (mayor en esta última), la venación secundaria de las pínnulas varía de doblemente dicotomizada en las pínnulas basales, hasta dividirse una sola vez en las apicales (Herbst, 1971). El borde de las pínnulas en el caso de $C$. mesozoica es uno de los caracteres más variables, polimorfismo que frecuentemente la presenta como 
muy similar a $C$. kurtzi, lo que lleva a preguntarse si definitivamente no se tratará de la misma especie.

\section{Género Todites Seward, 1900}

Especie tipo: T. williamsoni (Brongniart) Seward, 1900, Jurásico de Cayton, Yorkshire, Inglaterra.

Todites baldonii Herbst, 1988

Láms. 3, Fig. 15; Lám. 5, Fig. 25.

Material estudiado: STJU No. 62.

Procedencia: Cerro Calquinhue, Provincia de Concepción, Región del Biobío (Fig. texto 2; Tabla 1).

Discusión: como ya lo mencionaron Herbst et al.
(1998), Todites es un género poco frecuente en el Triásico de Sudamérica, por lo cual la comparación se remite a sólo dos especies, $T$. baldonii hallada en el Triásico Superior del Grupo El Tranquilo (Herbst, 1988) y T. chilensis en la Formación La Ternera (Herbst et al., 1998) también de la misma edad. Si bien los caracteres de los ejemplares estudiados coinciden con los dados por los autores para estas dos especies, existen evidentes afinidades en su morfología externa con Todites sp. descrita por Herbst (1988) para la flora triásica del Grupo El Tranquilo y con Todites baldonii en su arquitectura foliar, ya que esta última especie presenta una idéntica disposición general de las venas.

\section{CONCLUSIONES}

Las filicópsidas, al igual que el resto de la flora triásica gondwánica extratropical presenta un marcado exclusivismo, que se ha reflejado en la distribución localizada de ciertos taxones, generalmente restringidos al Gondwana sudoccidental. Las especies aquí estudiadas para el Triásico del Biobío son representantes de distintos grupos de estirpe paleozoica, que hallan su génesis en el Triásico y se difunden en el Mesozoico, o bien, aparecen, alcanzan su acmé y se extinguen durante el Triásico. El género Asterotheca, por ejemplo, tiene un amplio registro en Argentina, que va desde el Carbonífero hasta el Triásico Superior. Las especies del Triásico argentino (cinco especies) presentan un marcado endemismo (Zamuner et al., 2001), registrándose sólo dos especies fuera de Argentina, $A$. rigbyana Herbst, también presente en Chile y $A$. menendezii Herbst en Australia. La presencia en el Triásico del Biobío de la especie $A$. fuchsi (Zeiller) Herbst, exclusiva de los afloramientos chilenos, tal vez, se explique por una distribución restringida al borde costero del Gondwana occidental. Rienitsia, en cambio, apareció y se extinguió durante el Triásico. El género Dictyophyllum pertenece al grupo de filicópsidas que aparecen durante el Triásico y permanecen durante el Jurásico, al contrario de Chansitheca, grupo que ha quedado en el Triásico como taxón relictual del Pérmico. Esta última, junto a Gleichenites y Cladophlebis representan grupos que durante el
Triásico han tenido una amplia distribución geográfica. Algunas filicópsidas poseen un valor intrínseco como indicadores paleoclimáticos. Tal es el caso de las Marattiales, cuya presencia indicaría un clima cálido templado (Spalletti et al., 2003). Unido a ello, las Dipteridaceae indicarían un clima subtropical fuertemente estacional (Cantrill et al., 1995). Lo anterior viene a complementar un cuadro donde el Triásico del Biobío se ubica dentro del marco de los endemismos regionales, es decir, restringidos al Gondwana extratropical sudoccidental y, de endemismos locales, restringidos a los depocentros del margen protopacífico. Una fuerte evidencia apoya la idea de que las rocas sedimentarias del Triásico del Biobío se habrían depositado en proximidad del margen protopacífico: en primer lugar la existencia de estratos marinos entre niveles continentales, con abundantes invertebrados marinos (Pérez-Barría, 2004); en segundo lugar, la presencia de vastas extensiones de bosques de Linguifolium, que, como se había señalado, vinieron a reemplazar durante el Floriano (Triásico Tardío más alto) las comunidades dominadas por Dicroidium durante el Cortaderitiano (Triásico Medio a Cárnico). El acmé de las corystospermáceas durante el Cortaderitiano, en un sentido sinérgico, obedece a la existencia de condiciones de mayor aridez, es decir, un clima más seco y cálido durante el Cortaderitiano (Artabe et al., 2001) que durante el Floriano, donde la instauración de 
un clima megamonzónico y la influencia marina, permite la proliferación de las paleocomunidades dominadas por Linguifolium (Retallack, 1987; Spalletti et al., 1999, 2003) relegando a Dicroidium al estrato arbustivo, donde compite con Cladophlebis (Retallack, 1987). El endemismo en la costa del Protopacífico explica el fuerte vínculo florístico con la Formación La Ternera, al norte de Chile. A nivel de las filicópsidas, los estratos chilenos mejor estudiados (valle del río Biobío y La Ternera), presentan una alta afinidad entre ellos y, en ligero menor grado, con la Formación Paso Flores en el oeste argentino (Leppe, 2005).

Desde el punto de vista cronoestratigráfico, no se pueden utilizar los biocrones de filicópsidas pues éstos entregan edades poco acotadas, pero si se suman los otros taxa que representan la flora del Biobío, la presencia de eventos plutónicos en las secuencias que ligan el depocentro con los procesos extensionales de las proximidades del límite Triásico-Jurásico (Franzese y Spalletti, 2001), la presencia de comunidades boscosas subtropicales deciduas dominadas por Linguifolium y con declinación de las corystospermáceas (tercer evento florístico del modelo sinergístico de Spalletti et al., 2003), la presencia de un clima subtropical megamonzónico y el fuerte vínculo florístico con depocentros datados como próximos al límite $T / J$ (Leppe et al., 2003) apuntan fuertemente en el sentido de considerar a los estratos continentales del Triásico del Biobío como pertenecientes al Cárnico medio-Nórico superior del Gondwana extratropical sudoccidental, sobre la base de la superposición de los biocrones de 45 morfoespecies vegetales (Leppe, 2005), lo que reduce el rango a ca. 17 millones de años. Nielsen (2005), en cambio, sobre la base de la distribución cronoestratigráfica de 17 morfoespecies vegetales, ha postulado que los afloramientos del río Biobío serían de edad cárnica, restando importancia cronestratigráfica a los invertebrados.

\section{AGRADECIMIENTOS}

Los autores agradecen el apoyo prestado por los proyectos FONDECYT 2010105 'Paleobotánica del Triásico del valle inferior del río Biobío, Chile' y DIUC 'Análisis Estructural y Sedimentario de facies continentales del Triásico del Río Biobío'. Se agradece, asimismo, la ayuda gentil y desinteresada de una serie de colegas argentinos, entre los que se destaca a los Dres. A. Artabe (Museo de La Plata, Argentina) y R. Herbst (Instituto Superior de Geología-CONICET, Argentina). A su vez, al Dr. A.
Troncoso (Universidad de Talca, Chile) por sus importantes comentarios. Al Dr. A. Quinzio y al profesor R. Bonilla (Universidad de Concepción, Chile) por las facilidades brindadas para la recolección y reconocimiento de terreno. A los árbitros que contribuyeron con sus comentarios críticos a enriquecer y mejorar este manuscrito. Finalmente, a las señoras B. Oportus y J. Pacheco (Universidad de Concepción) por la valiosa edición realizada al texto preliminar.

\section{REFERENCIAS}

Abad, E. 2000. Análisis secuencial de facies en el Triásico del Biobío. In Congreso Geológico Chileno, No. 9, Actas 1: 424-427. Puerto Varas.

Abad, E.; Figueroa, J. 2003. Rocas volcánicas en el Triásico del Biobío: petrografía y marco geológico, cerro Calquinhue, VIII Región; Chile. In Congreso Geológico Chileno, No. 10, Actas: 8 p. Concepción.

Anderson, J.M.; Anderson, H.M. 1983. Palaeoflora of Southern Africa. Molteno Formation (Triassic) Gymnosperms. A.A. Balkema 2: $567 \mathrm{p}$.
Arrondo, O.; Morel, E.; Ganuza, D.; Hervé, F.; Parada, M.A.; Muehlebach, W. 1988. La Formación Panguipulli y su contenido paleoflorístico. In Congreso Geológico Chileno, No. 5, Actas 2: 137-145. Santiago.

Arrondo, O.; Spalletti, L.A.; Morel, E.; Ganuza, D. 1991. The sedimentological and paleobotanical characteristics of an Upper Triassic-Lower Liassic basin in northwestern Patagonia (Argentina). In Gondwana Seventh Proceedings (Ulbrich, H.; Rocha Campos, A.; editors). Instituto de Geociências, 
Universidade de Sao Paulo: 517-532. Sao Paulo.

Artabe, A.; Morel, E.; Spalletti, L.A. 2001. Paleoecología de las floras triásicas argentinas. In EI Sistema Triásico en la Argentina (Artabe, A.; Morel, E.; Zamuner, A.; editores). Fundación Museo La Plata Francisco $P$. Moreno: 199-225.

Artabe, A.; Morel, E.; Spalletti, L.A. 2002. Caracterización de las provincias fitogeográficas triásicas del Gondwana Extratropical. Ameghiniana 40 (3): $387-$ 405.

Ash, S. 1986. Fossil plants and the Triassic-Jurassic boundary. In The beginning of the age of dinosaurs: faunal change across the Triassic-Jurassic boundary (Pandian, K.; editor). Cambridge University Press: 21-30.

Azcárate, V.; Fasola, A. 1970. Sobre formas nuevas para la flora triásica de Los Molles. Boletín del Museo Nacional de Historia Natural (Chile) (29): 249-269.

Balme, B.; Helby, R. 1973. Floral modifications at the Permian-Triassic boundary in Australia. Canadian Society of Petroleum Geologists, Memoir 2: 433-444.

Belmar, M.; Morata, D. 2005. Nature and P-T-t constraints of very low-grade metamorphism in the TriassicJurassic basins, Coastal Range, central Chile. Revista Geológica de Chile 32 (2): 189-205.

Berchtold, F.; Presl, J.S. 1820. O. Prirozenosti Rostlin. J. Krause: 120 p. Prague.

Bonetti, M.; Herbst, R. 1964. Dos especies de Dyctyophyllum del Triásico de Paso Flores, Provincia del Neuquén, Argentina. Ameghiniana 3 (9): 273-279.

Brea, M. 2000. Paleoflora triásica de Agua de la Zorra, Uspallata, Provincia de Mendoza, Argentina: Lycophyta y Filicophyta. Ameghiniana 37 (2): 199204.

Brongniart, A. 1849. Tableau des genres de végetaux fósiles considerés sous le point de vue de leur classification botanique et de leur classification géologique. Dictionnaire Universel d'Histoire Naturelle 13:1-127. Paris.

Brüggen, J. 1950. Fundamentos de la Geología de Chile. Instituto Geográfico Militar: 374 p. Santiago.

Cantrill, D.; Drinnan, A.; Webb, J. 1995. Late Triassic plant fossils from the Prince Charles Mountains, east Antarctica. Antarctic Science 7 (1): 51-62.

Cleal, C.J.; Thomas, B.A. 1994. Paleozoic paleobotanic of Great Britain. Geological Conservation Review Series 9: $312 \mathrm{p}$.

Collinson, M. 1996. What use are fossil ferns? 20 years on: with a review of the fossil history of extant pteridophyte families and genera. In Pteridology in Perspective (Camus, J.; Gibby, M.; Jhons, R.; editors). Royal Botannic Garden: 349-394. Kew, London.

Corvalán, J. 1976. El Triásico y Jurásico de VichuquénTilicura y de Hualañé, Provincia de Cúrico. Implicaciones paleogeográficas. In Congreso Geológico Chileno, No. 1, Actas 1: A137-A154. Santiago.

Charrier, R. 1979. El Triásico en Chile y regiones adyacentes de Argentina: una reconstrucción paleogeo- gráfica y paleoclimática. Universidad de Chile, Departamento de Geología, Comunicaciones 26: 1-37.

Cucurella, J. 1978. Estudio geológico y radiométrico del valle inferior del río Biobío, Provincia de Concepción, VIII Región. Memoria de Título (Inédito), Universidad de Chile, Departamento de Geología: 342 p.

Dumortier, B. 1829. Analyse des Familles de Plantes. Casterman Jainé: 105 p.

Eldredge, N.; Gould, S.J. 1988. Punctuated equilibrium prevails. Nature (322): 211-212.

Franzese, J.; Spalletti, L.A. 2001. Late Triassic-early Jurassic continental extension in southwestern Gondwana: tectonic segmentation and pre-break-up rifting. Journal of South American Earth Sciences 14: 257-270.

Frenguelli, J. 1941. Las Camptopterídeas del Lías de Piedra Pintada en el Neuquén (Patagonia). Notas del Museo de la Plata, Paleontología 6 (27): 27-57.

Frenguelli, J. 1947. El género Cladophlebis y sus representantes en la Argentina. Anales del Museo de La Plata, Paleontología, sección B, Paleobotánica (2): 5-74.

Gallego, O.F.; Martins-Neto, R.G.; Nielsen, S.N. 2005. Conchostracans and insects from the Upper Triassic of the Biobío ('Santa Juana Formation'), south-central Chile. Revista Geológica de Chile 32 (2): 293-311.

Gradstein, F.M.; Ogg, J.G.; Smith, A.G.; Agterberg, F.P.; Bleeker, W.; Cooper, R.A.; Davydov, V.; Gibbard, P.; Hinnov, P.; House, M.R.; Lourens, L.; Luterbacher, H.P.; McArthur, J.; Melchin, M.J.; Robb, L.J.; Shergold, J.; Villeneuve, M.; Wardlaw, B.R.; Ali, J.; Brinkhuis, H.; Hilgen, F.J.; Hooker, J.; Howarth, R.J.; Knoll, A.H.; Laskar, J.; Monechi, S.; Powell, J; Plumb, K.A.; Raffi, I.; Röhl, U.; Sanfilippo, A.; Schmitz, B.; Shackleton, N.J.; Shields, G.A.; Strauss, H.; Van Dam, J.; Veizer, J.; van Kolfschoten, T.; Wilson, D. 2004. A Geologic Time Scale 2004. Cambridge University Press, 500 p.

Hallam, A.; Wignall, P. 1997. Mass extinctions and their aftermath. Oxford University Press: 320 p.

Herbst, R. 1963. Chansiteca argentina n. sp. del Triásico Superior de Santa Cruz, Patagonia. Ameghiniana 3 (4): 108-112.

Herbst, R. 1971. Paleophytologia Kurtziana II.7. Revisión de las especies argentinas del género Cladophlebis. Ameghiniana 8 (3-4): 265-281.

Herbst, R. 1977a. Sobre Marattiales (Filicopsidae) triásicas de Argentina y Australia. Parte I. El género Asterotheca. Ameghiniana: 14 (1-4): 1-18.

Herbst, R. 1977b. Sobre Marattiales (Filicopsidae) triásicas de Argentina y Australia. Parte II. Los géneros Danaeopsis y Rienitisia. Ameghiniana 14 (1-4): 1932.

Herbst, R. 1980. Flórula fósil de la Formación Los Patos (Sinemuriano) del río Los Patos, Provincia de San Juan, República Argentina. In Congreso Argentino de Paleontología y Bioestratigrafía, No. 2, Actas y Congreso Latinoamericano de Paleontología, No. 1: 
175-189. Buenos Aires.

Herbst, R. 1988. La flora triásica del grupo El Tranquilo, Provincia de Santa Cruz (Patagonia). Parte II. Filicopsida. Ameghiniana 25 (4): 365-379.

Herbst, R. 1992. Propuesta de clasificación de Dipteridaceae (Filicales) con un atlas de las especies de Argentina. D' Orbignyana 6: 1-71.

Herbst, R. 1996. New species of Gleichenites (Gleicheniaceae, Filicales) from the Upper Triassic of Argentina and Chile. Palaeobotanist 43 (3): 67-72.

Herbst, R. 2000. Dipteridaceae (Filicales) del Triásico Superior de Chile. Revista Geológica de Chile 27 (1): 65-81.

Herbst, R.; Melchor, R.; Troncoso, A. 1998. Las Pteridophyta y el paleoambiente de la parte media de la Formación La Ternera (Triásico Superior) en la quebrada La Cachivarita, III Región, Chile. Revista Geológica de Chile 25 (1): 85-107.

Herbst, R.; Troncoso, A. 1996. La tafoflora de Juan de Morales del Jurásico Medio (Formación Chacarilla), Región de Tarapacá, Chile. Revista Geológica de Chile 23 (1): 3-15.

Hervé, F.; Thiele, R.; Parada, M.A. 1976. Observaciones geológicas en el Triásico de Chile Central entre las latitudes 3530' y 4000' Sur. In Congreso Geológico Chileno, No. 1, Actas 1: A297-A313. Santiago.

Holser, W.; Schönlaub, H.; Boeckelmann, K.; Magaritz, M.; Orth, C. 1991. The Permian-Triassic of the Gartnerkofel-1 core (Carnic Alps, Austria): synthesis and conclusions. Abhandlungen der Geologischen Bundesanstalt 45: 213-232.

Knoll, A. 1984. Patterns of extinction in the fossil record of vascular plants. In Extinctions (Nitecki, M.H.; editor). University of Chicago Press: 23-68. Chicago.

Leppe, M. 2005. Paleobotánica del Triásico del valle inferior del río Biobío, Región del Biobío, Chile. Tesis de Doctorado (Inédito), Universidad de Concepción, Departamento de Botánica: $187 \mathrm{p}$.

Leppe, M.; Moisan, P. 2003. Nuevos registros de Cycadales y Cycadeoidales del Triásico Superior del río Biobío, Chile. Revista Chilena de Historia Natural 76 (3): 475-484.

Leppe, M.; Abad, E.; Palma-Heldt, S.; Moisan, P. 2003. La flora triásica del cerro Calquinhue, Región del BioBío, Chile. In Actas del Congreso Geológico Chileno, No. 10: 317-320. Concepción.

Lindley, J.; Hutton, W. 1834. The fossil flora of Great Britain: on figures and descriptions of vegetables remains found in a fossil state in this country. Carneby Editors 2: 208 p. London.

Morel, E.; Ganuza, D.; Zúñiga, A. 2000. Revisión paleoflorística de la Formación Paso Flores, Triásico Superior de Río Negro y del Neuquén, Argentina. Revista de la Asociación Geológica Argentina: 54 (4): 389-406.

Nielsen, S. 2005.The Triassic Santa Juana Formation at the lower Biobío river, south central Chile. Journal of Southamerican Earth Sciences 19: 547-562.
Pérez-Barría, L. 2004. Estudio taxonómico de la fauna de invertebrados del Triásico del Biobío, VIII Región, Chile): una aproximación preliminar. Reunión Anual de la Asociación Paleontológica Argentina. Ameghiniana 41 (4), Suplemento, Resúmenes: 18R.

Prezl, K.B. 1845. Gleicheniacedae. In Corda, A.J. 1845. Beiträge sur Flora der Vorwelt. S. 90, T.54: 128 p. Berlin.

Prantl, K. 1874. Ordo Marattiales. Lehrbuch der Botanik: 115-119

Regè, R. 1920. Note su alcuni vegetali del Carbonifero della Cina: Atti Società Italiani di Scienze Naturali di Milano 59 (3-4): 193-194.

Retallack, G. 1987. Triassic vegetation and geography of the New Zealand portion of the Gondwana supercontinent. In Gondwana Six; Stratigraphy and Paleontology (Elliot, D.; Collinson, J.; Mckenzie, G.; Haban, S.; editors). American Geophysical Union, Geophysical Monograph 41: 29-39.

Seward, A.C. 1900. Catalogue of Mesozoic Plants. The Jurassic Flora. Department of Geology of the British Museum (Natural History) 1: $86 \mathrm{p}$.

Seward, A.C. 1926. The Cretaceous plant-bearing rocks of western Greenland. Philosophical Transactions of the Royal Society of London 125B: 57-175.

Seward, A.C. 1969. Fossil Plants. Hofner Publishing Company 4: 543 p. New York and London.

Seward, A.C.; Dale, E. 1901. On the structure and affinities of Dipteris with notes on the geological history of the Dipteridinae. Philosophical Transactions of the Royal Society of London, Series B 194: 487-513.

Spalletti, L.A.; Artabe, A.; Morel, E.; Brea, M. 1999. Biozonación paleoflorística y cronoestratigrafía del Triásico Argentino. Ameghiniana 36: 419-451.

Spalletti, L.A.; Artabe, A.; Morel, E. 2003. Geological factors and evolution of Southwestern Gondwana Triassic Plants. Gondwana Research 6 (1): 119-134.

Spalletti, L.A.; Morel, E.M.; Artabe, A.E.; Zavattieri, A.M. Ganuza, D. 2005. Estratigrafía, facies y paleoflora de la sucesión triásica de Potrerillos, Mendoza, República Argentina. Revista Geológica de Chile 32 (2): 249272.

Steinmann, G. 1921. Rhätische Floren und Landverbindungen auf der Südhalbkugel. Geologische Rundschau 11: 350-354.

Stewart, W.N. 1983. Paleobotany and the evolution of plants. Cambridge University Press: 405 p.

Stipanicic, P.; Menéndez, C. 1949. Contribución al conocimiento de la flora fosil de Barreal (Provincia de San Juan). 1. Dipteridaceae. Boletín de Informaciones Petroleras 291: 44-73. Buenos Aires.

Suárez, M.; Bell, C.M. 1992. Triassic rift-related sedimentary basins in northern Chile (240-29'S). Journal of South American Earth Sciences 6 (1): 109-121).

Tanai, T. 1981. Geological notes on the fossil localities. In Paleobotanical Survey to Southern Chile (Nishida, M.; editor), Report, Faculty of Science Chiba University: 10-30. 
Tanner, L. 2000. Palustrine-lacustrine and alluvial facies of the (Norian) Owl Rock Formation (Chinle Group), Four Corners Region, southwestern U.S.A.: implications for Late Triassic paleoclimate. Journal of Sedimentology Research 70: 1280-1289.

Tavera, J. 1960. El Triásico del valle inferior del río Biobío. Universidad de Chile, Facultad de Ciencias Físicas y Matemáticas, Anales (17): 321-349.

Thiele, R. 1965. El Triásico y el Jurásico del Departamento de Curepto en la Provincia de Talca. Universidad de Chile, Departamento de Geología, Publicación (28): $46 \mathrm{p}$.

Thiele, R.; Hervé, F.; Parada, M.A. 1976. Bosquejo geológico de la isla Huapi, lago Ranco, Provincia de Valdivia: contribución al conocimiento de la Formación Panguipulli (Chile). In Congreso Geológico Chileno, No. 1, Actas 1: A115-A136. Santiago.

Tidwell, W.; Ash, S. 1994. A review of selected Triassic to Early Cretaceous ferns. Journal of Plant Research 107: 417-442.

Traverse, A. 1988. Plant evolution dances to a different beat; plants and animal evolutionary mechanisms compared. Historical Biology 1: 277-302.

Troncoso, A.; Herbst, R. 1999. Ginkgoales del Triásico del norte de Chile. Revista Geológica de Chile 26 (2): 255-273.

Walkom, A. 1932. Fossil plants from Mount Piddington and Clarence Siding. Proceedings of the Linnean Society of New South Wales 57 (3-4): 123-126.

Wang, G.; Guignard, G.; Barale, G. 1999. Morphological and ultrastructural studies on spores in situ of Oligocarpia (Gleicheniaceae) from the Lower Permian of Xinjiang, China. International Journal of Plant Sciences 160 (5): 1035-1045.

Webb, J. 1982. Triassic species of Dictyophyllum from eastern Australia. Alcheringia 6: 79-91.

Yao, X. Taylor, T. 1988. On a new gleicheniaceous fern from the Permian of South China. Review of Paleobotany and Palinology 54: 121-134.

Zamuner, A.; Zavattieri, A.; Artabe, A.; Morel, E. 2001. Paleobotánica. In El Sistema Triásico en la Argentina (Artabe, A.; Morel, E.; Zamuner, A.; editores). Fundación Museo La Plata Francisco P. Moreno: 143-184.

Zavattieri, A.M.; Herbst, R.; Muñoz, J. 2003. Microflora de la Formación Panguipulli (Triásico Superior), en Licán Ray, Lago Calafquén, X Región, Chile. Ameghiniana 40 (4): 585-600.

Ziegler, A.; Parrish, J.M.; Yao, J.; Gyllenhaal, E.; Rowley, D.; Parrish, J.T.; Nie, S.; Bekker, A.; Hulver, M. 1994. Early Mesozoic phytogeography and climate. In Palaeoclimates and their modeling (Allen, J.; Hoskins, B.; Sellwood, V.; Spicer, V.; Valdés, P.; editors). Chapman y Hall: 89-98. London. 
LÁMINAS 1-5 


\title{
LÁMINA 1
}

(Escala en centímetros)

Figuras 1-2

\section{Asterotheca rigbyana rigbyana Herbst, 1977}

p. 87

Cerro Calquinque, camino a Buenutaqui, Provincia de Concepción, Región del Biobío (Fig. texto 2; Tabla 1). Triásico Superior.

1. Fronda fértil.

2. Fronda estéril bipinnada.

\section{Figura 3}

\section{Asterotheca fuchsi (Zeiller) Herbst, 1998}

p. 88

Patagual y Cerro Calquinque, Provincia de Concepción, Región del Biobío (Fig. texto 2; Tabla 1). Triásico Superior.

3. Frondas, fértil (a) y estéril (b) .

Figura 4

\author{
Rienitsia colliveri Herbst, 1977 \\ p. 88
}

Cerro Calquinque, Patagual y Mardoñal, Provincia de Concepción, Región del Biobío (Fig. texto 2; Tabla 1. Triásico Superior.

4. Fronda estéril.

Figura 5

Dictyophyllum (Dictyophyllum) tenuifolium (Stipanicic y Menéndez) Bonetti y Herbst, 1964 p. 89

Santa Juana, vía férrea hacia Gomero, camino a Quilacoya, Cerro Calquinhue, Patagual, Mordañal y Quebrada Calcura, Provincia de Concepción, Región del Biobío (Fig. texto 2; Tabla 1). Triásico Superior.

5. Fragmento de pinna. 
LÁMINA 1
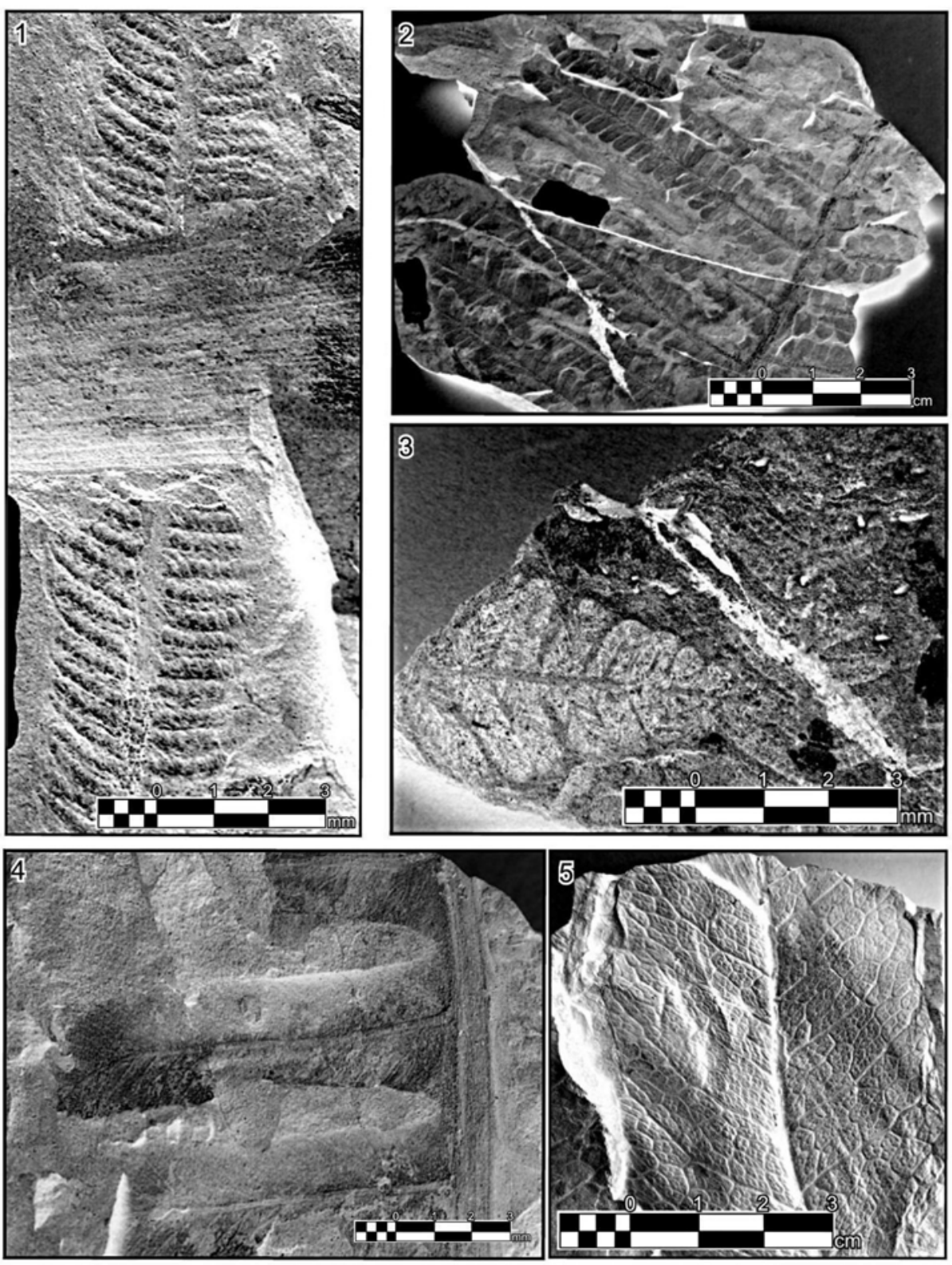


\section{LÁMINA 2}

(Escala en centímetros)

Figuras 6-7

Dictyophyllum (Thaumatopteris) rothi Frenguelli, 1941

p. 89

Buenuraqui, Talcamávida, Gomero y cruce Rere, Provincia de Concepción, Región del Biobío (Fig. texto 2; Tabla1). Triásico Superior.

6. Porción de pinna.

7. Porción de fronda estéril.

Figura 8

\section{Dictyophyllum (Dictyophyllum ) fuenzalidai Herbst, 2000}

p. 89

Santa Juana, Provincia de Concepción, Región del Biobío (Fig. texto 2; Tabla 1). Triásico Superior.

8. Porción media de pinna

Figuras 9-11

Gleichenites quilacoyensis Leppe y Moisan sp. nov.

p. 90

Cerro Calquinque, Provincia de Concepción, Región del Biobío (Fig. texto 2; Tabla 1). Triásico Superior.

9. Holotipo STJU No. 2. Porción media de fronda estéril.

10 y 11. Detalle de la forma y disposición de las pínnulas estériles (10), fértiles (11). 
LÁMINA 2
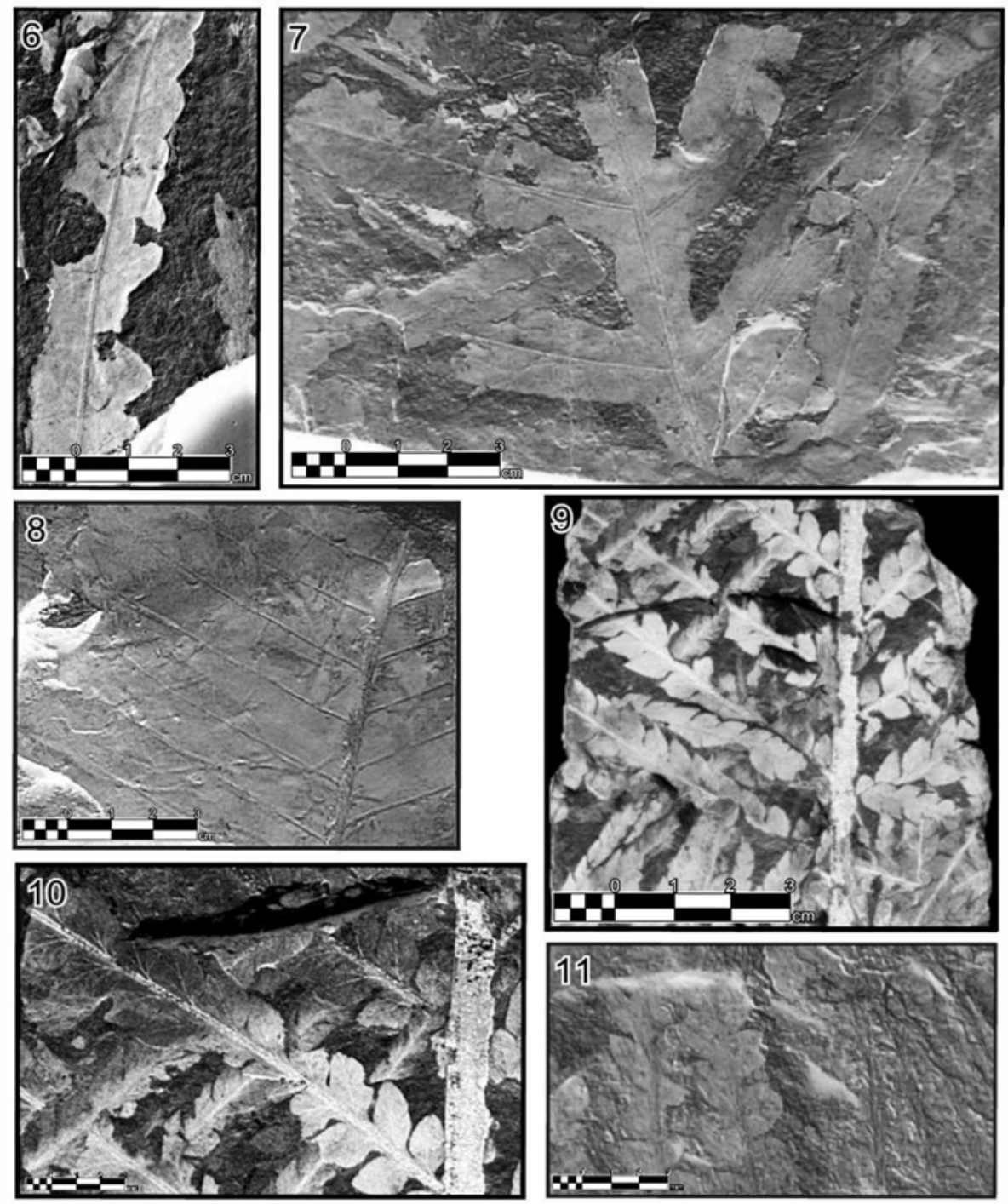


\section{LÁMINA 3}

(Escala en centímetros)

Figuras 12-13

\section{Chansitheca argentina Herbst, 1963}

$$
\text { p. } 91
$$

Cerro Calquinhue, Gomero, camino a Buenuraqui, Provincia de Concepción, Región del Biobïo (Fig. texto 2; Tabla 1). Triásico Superior.

12. Porciones de frondas estériles y fértiles.

13. Detalle de pínnulas fértiles.

Figura 14

\section{Cladophlebis kurtzi Frenguelli. 1947}

$$
\text { p. } 91
$$

Santa Juana y Cerro Calquinhue, Provincia de Concepción, Región del Biobío (Fig. texto 2; Tabla 1). Triásico Superior

14. Porción apical.

Figura 15

Todites baldonii Herbst, 1988

p. 92

Cerro Calquinhue, Provincia de Concepción, Región del Biobío (Fig. texto 2; Tabla 1). Triásico Superior.

15. Fronda estéril bipinnada. 

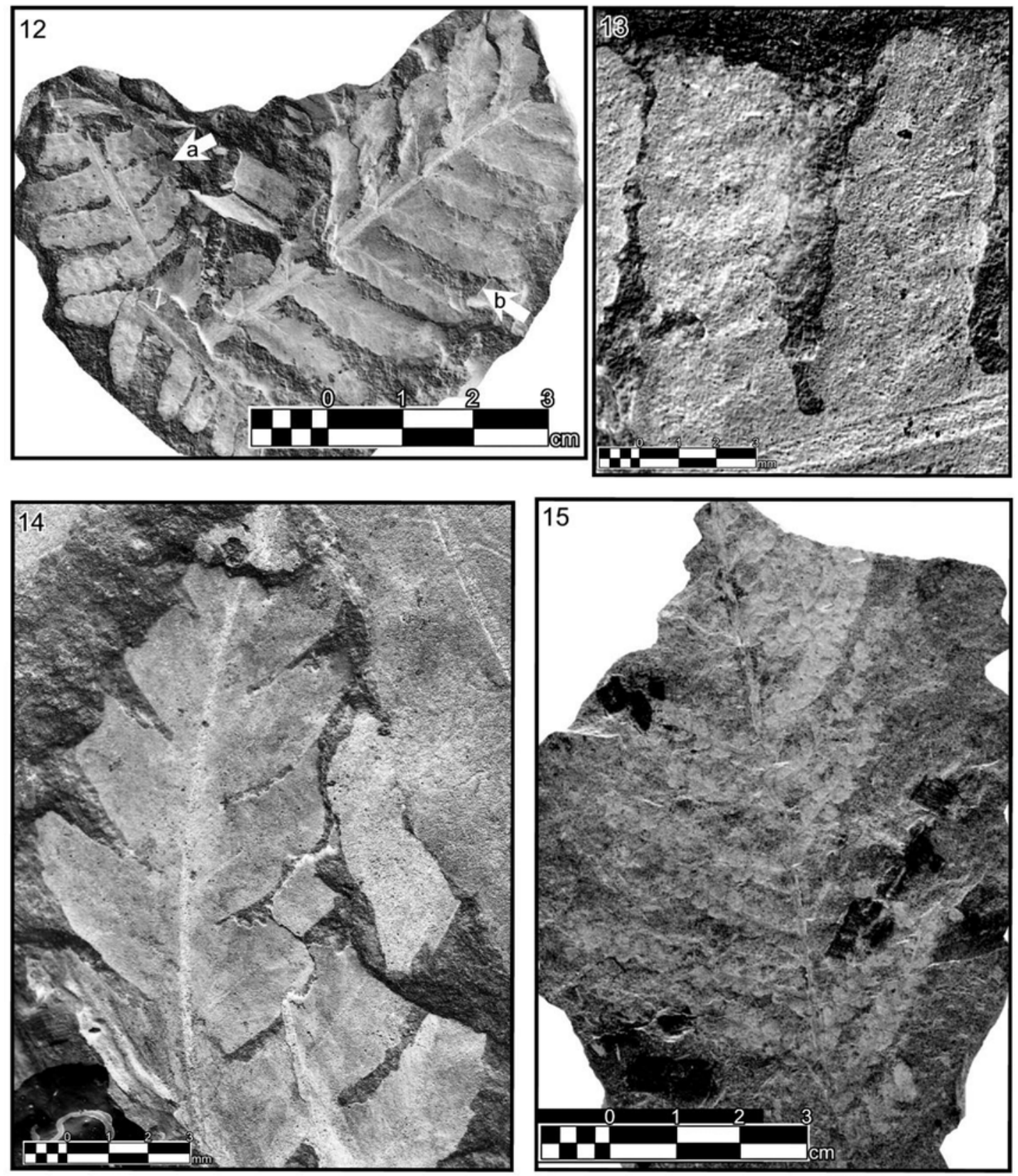


\section{LÁMINA 4}

(Escala en centímetros)

Figura 16

\section{Figura 17}

Figura 18

Figura 19

Figura 20

Figura 21

\section{Asterotheca rigbyana rigbyana Herbst, 1977}

p. 87

Cerro Calquinhue, Provincia de Concepción, Región del Biobío (Fig. texto 2; Tabla 1). Triásico Superior.

16. Esquema de la disposición y número de los sinangios y la venación simple.

\section{Asterotheca fuchsi (Zeiller) Herbst, 1968}

p. 88

17. Venación secundaria dicotomizada y disposición y número de los sinangios.

\section{Rienitsia colliveri Herbst, 1968}

p. 88

Cerro Calquinhue, Patagual y Mardoñal, Provincia de Concepción, Región del Biobío (Fig. texto 2; Tabla 1). Triásico Superior.

18. Esquema de fronda estéril.

\section{Dictyophyllum (Dictyophyllum) tenuifolium (Stepanicic y Menéndez) Bonetti y Herbst, 1964} p. 89

Santa Juana, vía férrea hacia Gomero, camino a Quilacoya, Cerro Calquinhue, Patagual, Mardoñal, Quebrada Colcura, Provincia de Concepción, Región del Biobío (Fig. texto 2; Tabla 1). Triásico Superior.

19. Detalle de la venación reticulada.

Dictyophyllum (Dictyophyllum) fuenzalidai Herbst, 2000

p. 89

Santa Juana, Provincia de Concepción, Región del Biobío (Fig. texto 2; Tabla 1). Triásico Superior.

20. Esquema de pinna y venación.

\section{Dictyophyllum (Thaumatopteris) rothi Frenguelli, 1941 \\ p. 89}

Buenuraqui, Talcamávida, Gomero, Cruce Rere, Provincia de Concepción, Región del Biobío, (Fig. texto 2; Tabla 1). Triásico Superior.

21. Detalle de la vena media y las venas secundarias y terciarias en un patrón reticulado. 

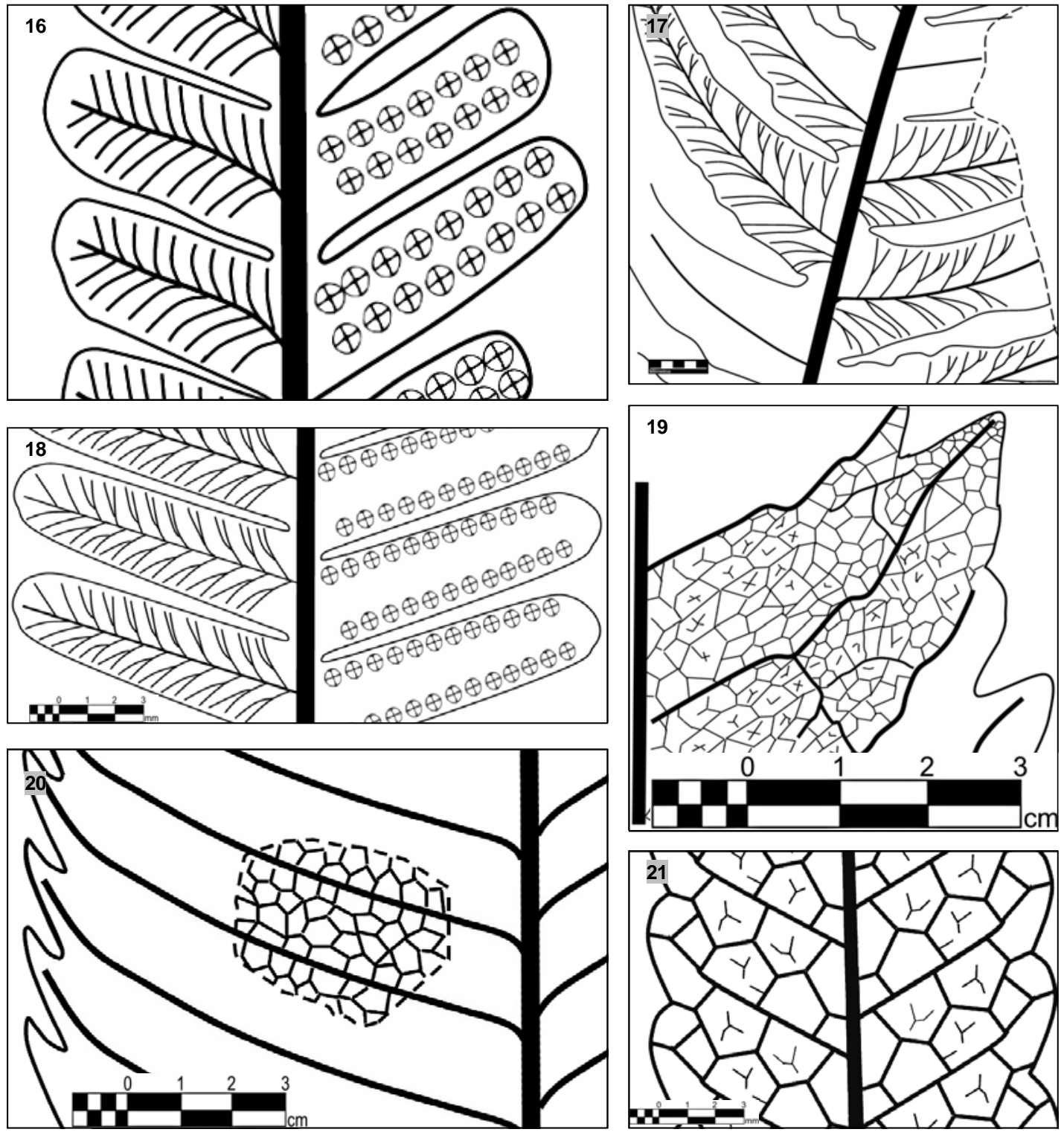


\section{LÁMINA 5}

(Escala en centímetros)

Figura 22

Gleichenites quilacoyensis Leppe y Moisan sp. nov.

p. 90

Cerro Calquinhue, Provincia de Concepción, Región del Biobío (Fig. texto 2; Tabla 1). Triásico Superior.

22. Esquema de pínnulas estériles y fértiles del Holotipo STJU No 2.

Figura 23

Chansitheca argentina Herbst, 1963

p. 91

Cerro Calquinhue, Gomero, camino a Buenuraqui, Provincia, de Concepción, Región del Biobío (Fig. texto 2; Tabla 1). Triásico Superior.

23 Esquema de la agrupación de los soros y detalle de la venación.

Figura 24 Cladophlebis kurtzi Frenguelli, 1947

p. 91

Santa Juana, Cerro Calquinhue, Provincia de Concepción, Región del Biobío, (Fig. texto 2; Tabla 1). Triásico Superior.

24. Esquema de las pínnulas.

Figura 25

\section{Todites baldonii Herbst, 1988}

p. 92

Cerro Calquinhue, Provincia deConcepción, Región del Biobío (Fig. texto 2; Tabla 1). Triásico Superior.

25. Esquema de las pínnulas y venación. 

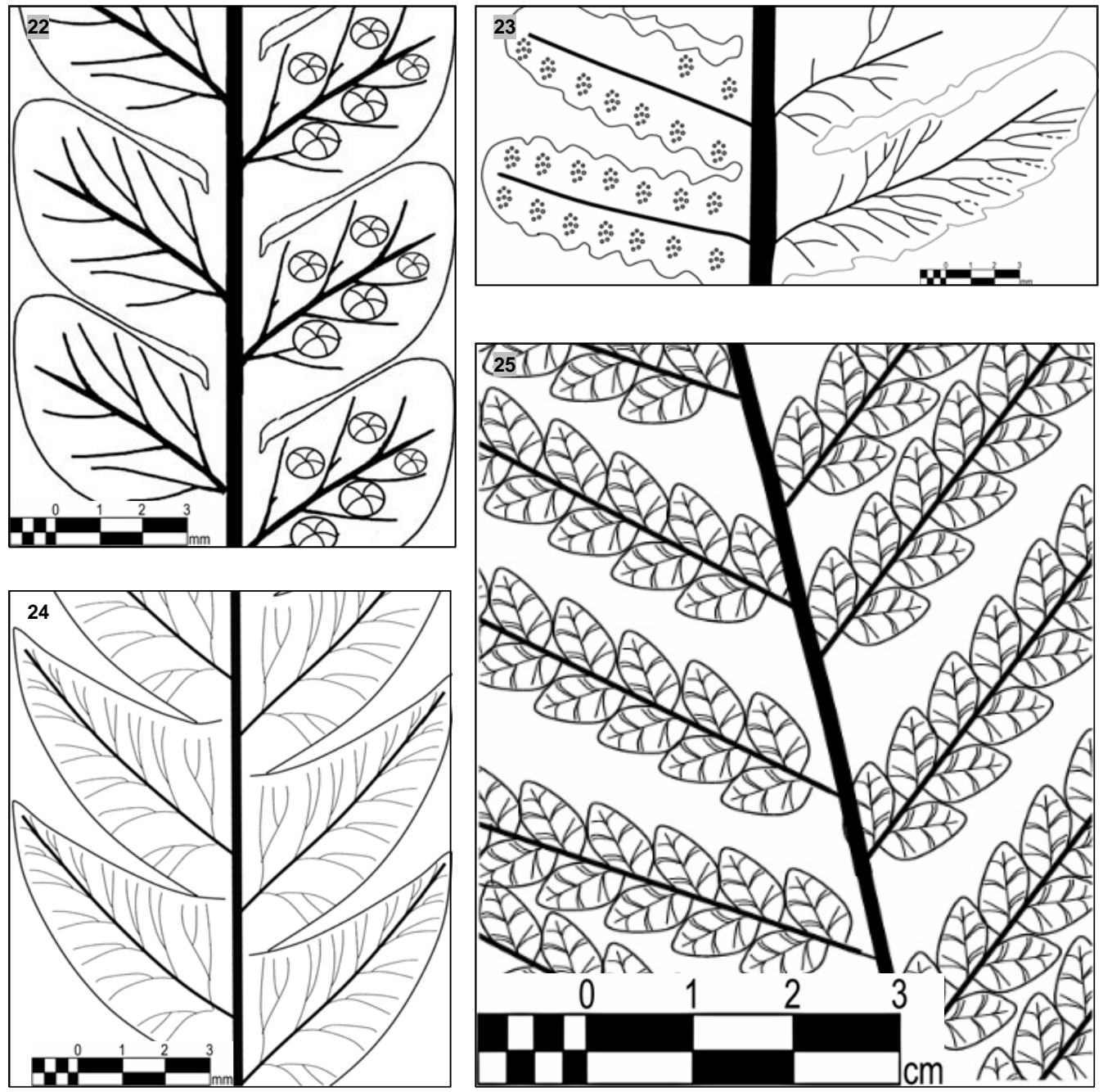